\title{
Jump and free fall! Memory, attention, and decision-making processes in an extreme sport
}

\author{
Judit Castellà (D), Jaume Boned, Jorge Luis Méndez-Ulrich (iD and Antoni Sanz \\ Department of Basic, Developmental, and Educational Psychology, Autonomous University of Barcelona, Barcelona, Catalonia
}

\begin{abstract}
In the present study, we explored the effects of high arousal on cognitive performance when facing a situation of risk. We also investigated how these effects are moderated by either positive or negative emotional states (valence). An ecological methodology was employed, and a field study was carried out in a real-life situation with 39 volunteer participants performing a bungee jumping activity and a control group of 39 participants. Arousal and valence were assessed with the Self-Assessment Manikin (SAM). Working memory capacity (reverse digit span), selective attention (Go/No-Go task) and decision-making (lowa Gambling Task) were assessed at 3 time points: $30 \mathrm{~min}$ before the jump, immediately after the jump, and approximately $8 \mathrm{~min}$ after the onset of the previous phase. The results indicate that high arousal accompanied by high positive valence scores after jumping either improved performance or led to a lack of impairment in certain cognitive tasks. The Processing-Efficiency and the Broaden-and-Build theories are put forward to explain emotional moderation of cognitive performance in potentially life-threatening situations.
\end{abstract}

ARTICLE HISTORY

Received 28 September 2018

Revised 12 March 2019

Accepted 6 May 2019

\section{KEYWORDS}

Arousal; valence; cognitive processing; bungee jumping
In laboratory settings, a large body of research has demonstrated that cognitive processing is influenced by affective state and it has been argued that high arousal provokes deficits in attentional control, which leads to impairments in performing tasks that involve the central executive of working memory / prefrontal cortex (Arnsten, 2009; Eysenck, Derakshan, Santos, \& Calvo, 2007). However, one obvious problem that arises when testing these relationships in the laboratory is that intense emotional responses cannot be artificially induced for ethical and practical reasons. In this regard, there is a lack of empirical data that ascertains the mechanisms underpinning emotion and cognition in real-life situations that intrinsically elicit a high emotional response, and thus there is a need to add ecological validity to cognition studies (Brewer, 2000; Ruffman, 2011; Shadish, Cook, \& Campbell, 2002).

To our knowledge, only a few studies have explored the impact of affective response on cognitive abilities in real-life contexts. For instance, Idzikowski and Baddeley (1987) tested parachutists on a range of cognitive tasks prior to entering the aircraft. A decline in almost all measures was found, which was interpreted in terms of impairment in the functioning of the central executive component of working memory (Baddeley, 1986, 2007; Baddeley \& Hitch, 1974). Leach and Griffith (2008) administered a task that involved both storage and processing components of working memory, to novice and expert parachutists. However, unlike Idzikowski and Baddeley, participants were also tested immediately after landing. Novice and experienced parachutists showed a decrease in performance in both components of the task prior to the jump, but in the landing phase novice participants performed significantly worse while experienced parachutists showed full recovery of the storage component. These results differ somewhat from those found by Robinson, Sünram-Lea, Leach, and Owen-Lynch (2008), since participants undergoing an evacuation from a submerged and inverted helicopter who were 
administered a similar task showed no impairment prior to task exposure, but performance was significantly reduced following stress exposure. In another study (Robinson, Leach, Owen-Lynch, \& Sünram-Lea, 2013), participants who underwent a fire-fighting emergency simulation also reported impairments in visual memory when tested immediately after the exercise. Finally, British Royal Air Force aircrew members participating in a military survival exercise were given various attentional tasks and showed a significant decrease in selective and sustained attention, suggesting an impairment in the ability to hold active memory representations in the presence of interference (Leach \& Ansell, 2008).

Taken together, the studies conducted in real-life environments with powerful stressors seem to have yielded mixed results. However, the vast majority mainly report impairments before, and particularly after, exposure to a highly arousing situation on a range of cognitive tasks. A theory that can be put forward to explain this decline is the Processing Efficiency Theory (Eysenck et al., 2007; Eysenck \& Calvo, 1992). The theory aims to explain how anxiety impacts performance and assumes that worry is activated under stressful situations, which causes interference by consuming the limited resources of the storage and processing components of working memory, which in turn enhances the use of auxiliary strategies to minimise the negative state of anxiety. A recent reformulation (Eysenck \& Derakshan, 2011) includes the ideas that (1) anxiety reduces the focus of attention to threat-related stimuli, and (2) inhibition and shifting functions of the central executive become impaired. However, this theory would predict no differences in performance after exposure to the stressor because under stressful circumstances working memory capacity is taken up with negative thoughts related to the task at hand, which are supposed to cease after exposure. Nevertheless, many of the cited studies have reported a detrimental effect on performance after experiencing a high arousal or extreme situation (Leach \& Ansell, 2008; Robinson et al., 2008, 2013). One possible explanation is that participants may have some residual anxiety and so their resources for information processing have not been restored to baseline levels immediately after exposure, particularly if the arousing activity involves an acute increase in activation or if the participants are not experts and are therefore unable to rapidly decrease their anxiety and the negative outcomes of worry.
In our study, we wanted to explore these dynamics in a situation that produces a peak in arousal, lasting less than one minute in total, and, unlike previous studies has the particularity of being a leisure activity. Therefore, we recruited participants who voluntarily took part in a bungee jumping session, which involves jumping from a high bridge with a long elastic rope tied to a full-body harness, so that the participant is pulled back by the rope before hitting the ground. Only novice jumpers were tested since previous studies in jumpers such as parachutists have shown that levels of expertise can markedly affect selfreported arousal, physiological measures (Fenz \& Epstein, 1967) and the impact of these variables on cognitive abilities (Idzikowski \& Baddeley, 1987). Hennig, Laschefski, and Opper (1994) conducted a study on novice bungee jumpers and found higher subjective ratings of anxiety before the jump, which rapidly dropped thereafter, and higher levels of salivary cortisol after the jump, which returned to baseline within an hour. Interestingly, following the jump, a $200 \%$ increase in $\beta$-endorphin was found, which correlated with euphoria ratings. They also found that blood pressure and heart rate were markedly affected by the stressful nature of the activity. Taken together, these findings suggest that bungee jumping could be an optimal setting for conducting research on how emotion could affect cognitive processes in a real-life environment.

Moreover, the characteristics of the activity allows for exploring not only subjective arousal, but also valence, which might be positive and could have the potential to moderate the negative effects of anxiety on cognition predicted by the processing efficiency theory. Valence is another fundamental dimension of affective states (Bradley \& Lang, 1994; Russell, 1980) and its interaction with arousal has proven to be key in reported distortions in cognitive processes such as size perception (Geuss, Stefanucci, de BenedictisKessner, \& Stevens, 2010), colour perception (Ziems \& Christman, 1998), temporal perception (Angrilli, Cherubini, Pavese, \& Manfredini, 1997; Castellà, Cuello, \& Sanz, 2017), or motor planning (Coombes, Higgins, Gamble, Cauraugh, \& Janelle, 2009). Regarding the cognitive tasks used in the present study, working memory was assessed due to its relevant role in complex cognitive abilities (Baddeley, Gathercole, \& Papagno, 1998; Jonides, 1995; Salthouse, 1992). Selective attention was also tested due to its critical role in daily life performance and its relationship with working memory processes (Awh, Vogel, \& 
Oh, 2006; Engle, Kane, \& Tuholski, 1999). In addition to these two basic skills that play an important role in normal cognitive functioning, we also assessed the high-level process of decision-making, which is determined by emotion (Damasio, 1998). If these capacities are distorted or interrupted under conditions of high arousal, the individual's ability to respond and react might be compromised, leading to negative consequences in situations where life is at risk. However, the positive valence associated with the activity might play a role that is worth exploring and represents a novel aspect of the present study. With this purpose in mind, several cognitive tasks were administered to a group of bungee jumpers before the jump, immediately after the jump, and at a third time point after the jump. On the basis of the processing efficiency theory and previously reported findings, we expected to find impaired cognitive performance immediately after the jump in comparison with a control group. However, valence might have the potential to moderate the negative impact of high arousal (Angrilli et al., 1997; Castellà et al., 2017).

\section{Methods}

\section{Participants}

The original sample consisted of 80 participants, 41 of which were individuals attending a bungee jumping session and that consented to voluntarily take part in the study after being informed about the procedure. The control group consisted of a group of 39 participants with similar characteristics in terms of age and gender. This group performed the tasks following the same procedure as the experimental group, and were recruited and assessed in a similar environment but without experiencing any arousing activity between phases. Thus, the whole sample was incidental and recruited in situ. Two participants were excluded because they failed to complete the questionnaires and/or correctly carry out the online tasks. The final sample therefore consisted of 78 participants, 39 males (50\%) and 39 females (50\%). In the jump group $56 \%$ were male and $44 \%$ female, ranging from 18 to 39 years old $(M=24.8, S D=5.59)$, and in the control group 56\% were female and $44 \%$ male, ranging from 18 to 33 years old $(M=22.3, S D$ $=3.29$ ). Of the jump group, $90.3 \%$ of the participants had no prior experience of bungee jumping. Additional mean comparison analyses revealed that there were no significant differences in any of the obtained measures as a function of gender. Moreover, age did not significantly correlate with any of the variables. Therefore, these two socio-demographic variables were not subject to any further analysis as independent variables.

\section{Materials}

The experimenters carried an identifier so that participants could recognise them as researchers from the Autonomous University of Barcelona conducting a psychological study on high-risk sports. A questionnaire designed in Google Forms and synchronised in real time with a Google Spreadsheet was administered using smartphones with a 5.8-inch screen in each of the three phases. The questionnaire included the Self-Assessment Manikin test (SAM; Bradley \& Lang, 1994), which is a pictorial assessment instrument that addresses the dimensional analysis of affective states. The participants were presented with the two sets of pictograms for each of the two affective dimensions (arousal and valence), and were asked to assess their current state on a Likert scale ranging from 1 to 9. The questionnaires also included a memory span task, and socio-demographic variables (age, sex, and previous experience in bungee jumping or a related activity). Working memory was assessed by a reverse digit span task that consists of encoding an increasing series of digits (from 3 to 6 items) and retrieving them verbally in the reverse order, thereby involving storage and processing of verbal information. Two measures were taken: span - defined as the maximum list length that the participant could correctly recall in reverse order for at least 1 out of the 2 series of a given span - and a less strict measure, that is, the total number of correct series recalled without stopping the scoring when they failed to recall the two series of a given span.

In addition to the questionnaire, the participants performed 2 online experimental tasks on smartphones: A Go/No-Go task, to assess selective attention, and the lowa gambling task to assess decisionmaking. The tasks were performed using Presentation $^{\circledast}$ (2018), more specifically, Psych Lab 101 software, which is a platform developed by this company and contains several cognitive psychology experiments especially designed to be run on smartphones, adapted to touch responses, and available for Android and IOS devices. In the Go/No-Go task participants had to respond by touching the screen (Go) every time a letter A, B, or C appeared, and withhold 
responses (No-Go) when an $\mathrm{X}$ was shown. There were 72 trials (48 Go and 24 No-Go, randomly presented), and items were presented successively for $1000 \mathrm{~ms}$ with an ISI of 700 ms. Reaction time (RT) and accuracy were recorded. The lowa Gambling Task used was based on the original task designed by Bechara, Damasio, Damasio, and Anderson (1994). This consisted of 50 trials using four decks of cards. The participants had to choose from the decks with the objective of winning as much as possible without being consciously aware of the reward/penalty properties of each deck. Two decks had small rewards and penalties, but led to a net gain over time whilst the other two decks carried large rewards and penalties but led to a net loss over time. These specific tasks were chosen for being paradigmatic in working memory, attention, and decision making research, and because they were sufficiently short to capture the impact of emotional responses.

\section{Design and procedure}

The study was carried out following the ethical standards for human research of the Ethical Committee of the Autonomous University of Barcelona. The procedure for the jump group consisted of three phases, which took place at different times: Phase 1: Approximately half an hour before jumping, Phase 2: immediately after the jump, and Phase 3: approximately $8 \mathrm{~min}$ after the onset of the previous phase. The procedure used for the control group was identical in terms of the timing of the phases and tasks, and it was carried out in a very similar environment (outdoors, with a certain degree of social interaction and noise, at 3 different times), the difference being that they did not engage in any arousing or emotional activity between phases, and therefore no emotional changes were expected to occur between these phases. The resulting design was a 3 (phase as a within-subjects factor) $\times 2$ (group as a between-subjects factor) quasi-experimental design.

Data was collected for the jump group at the bridge (see Figure 1) where the bungee jumping activity was scheduled (Castellbell i el Vilar, Catalonia; UTM coordinates: N 41 ${ }^{\circ} 37.39^{\prime}$ E $1^{\circ} 51.299^{\prime}$ ) in 3 different sessions organised by La Sargantana, a local enterprise that specialises in risky sports such as climbing, canyoning, and bungee jumping, and which agreed to participate in this study. The enterprise is run by certified mountain guides who are authorised by current legislation and follow the standard security protocols. The experimenters met the jumpers before starting the activity, informed them about the research, and asked for permission to voluntarily take part. After the first questionnaire and tasks had been administered (Phase 1, which lasted around $8 \mathrm{~min}$ ), the participants were identified with a code so that their anonymous data could be matched with those collected in the subsequent phases. They then proceeded to listen to the team of guides who instructed them on how to jump safely. Two experts monitored the jump, and the rope was tied to a full-body harness. The height of the bridge was $30 \mathrm{~m}$ and the total free fall was $15 \mathrm{~m}$, followed by a pendulum movement for approximately $25 \mathrm{~s}$ until touching the ground; thus the whole episode lasted around half a minute. At approximately 30 min after the first phase, Phase 2 then took place immediately after the jump. The experimenter approached each participant at the jump reception point as they were removing the harness and the questionnaire and tasks were administered again. Phase 2 was followed by Phase 3 , which started approximately 8 min after the onset of Phase 2, and consisted of repeating the whole procedure. Note that digits in the reverse digit span task were different in each phase but counterbalanced across participants. The SAM test was always administered at the beginning of each testing phase but the order of the 3 cognitive tasks was counterbalanced to minimise learning and order effects, so that in each experimental session, a certain order was used following a Latin-square design (Span-Go-lowa, Go-lowa-Span, lowa-Span-Go).

\section{Results}

At first glance, the scores obtained in each phase for the affective variables, i.e. the means for arousal and valence, were higher in the jump group compared with the control group in all of the phases. A oneway ANOVA revealed that these differences were statistically significant for both parameters in all the phases, except for the arousal score in Phase 3, indicating that the task (jumping) was able to induce acute changes in both variables, increasing valence, but particularly arousal. Table 1 summarises the mean scores of arousal and valence in each group and the significance of the comparison between groups.

The pattern of results obtained for both parameters and the significant differences between phases in the two groups are graphically represented in Figures 2 and 3. A significant interaction between phase and group was found for arousal $(F(2,148)=33.31$, 


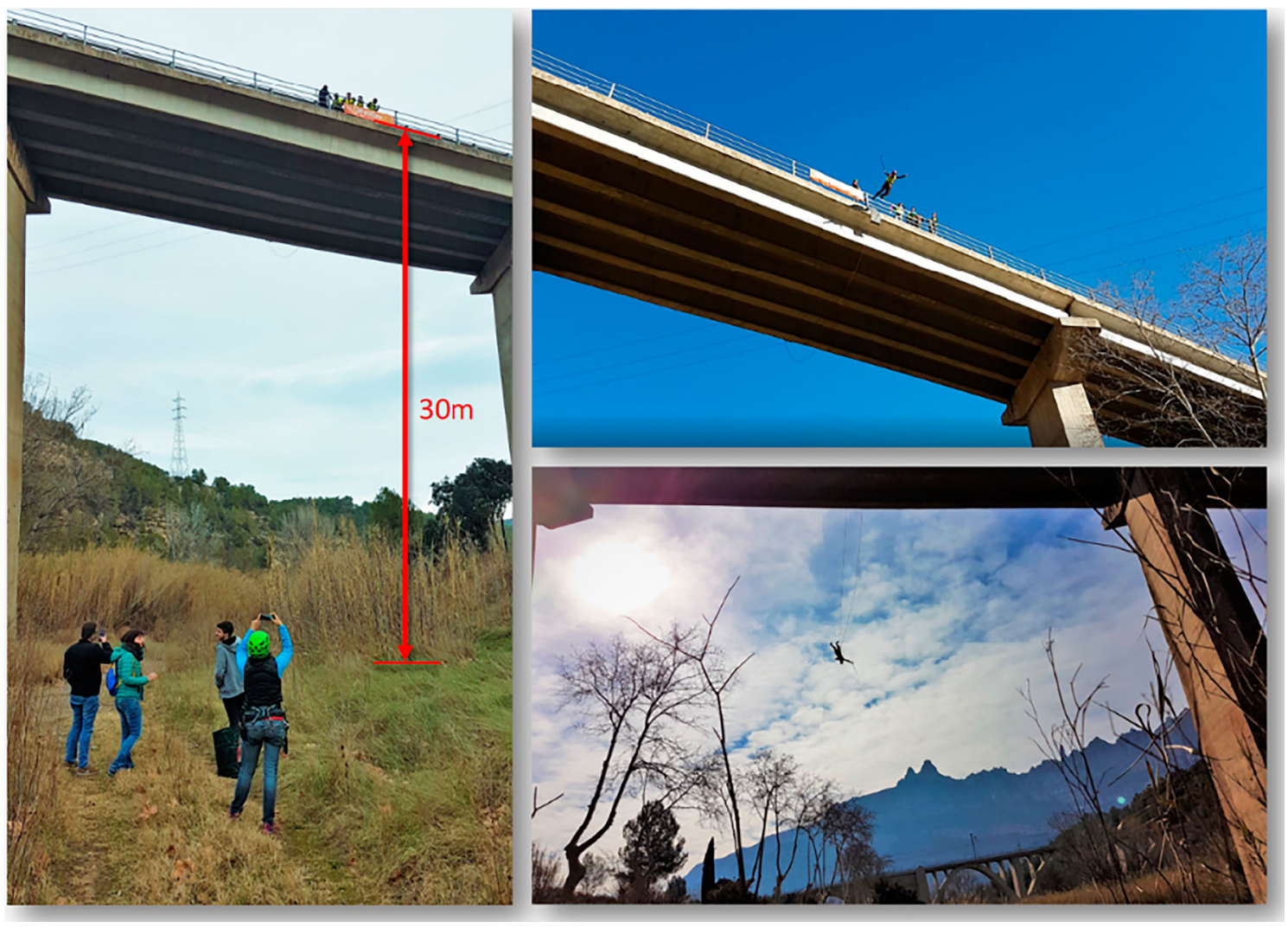

Figure 1. Photographic images of the study settings. General view of the bridge, experimenters, and participants during a jump.

$p<.001$, partial $\left.\eta^{2}=.31\right)$ and valence $(F(2,138)=10.01$, $p<.001$, partial $\eta^{2}=.13$ ). In the jump group, the contrasts (simple method) of the within-subjects linear model showed that the changes in arousal were statistically significant between Phase 1 and Phase 2 $\left(F(1,39)=87.07, \quad p<.001\right.$, partial $\left.\eta^{2}=.69\right)$, and between Phase 2 and Phase $3(F(1,39)=75.11$, $p<.001$, partial $\eta^{2}=.66$ ). No significant differences

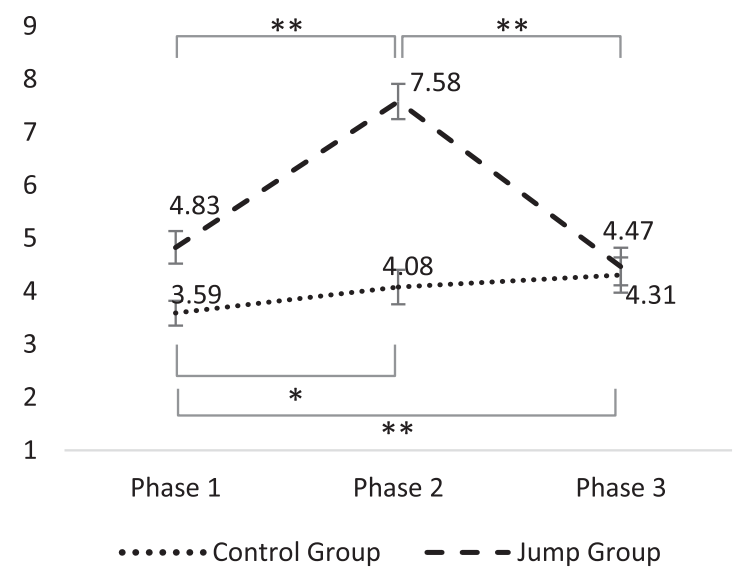

Figure 2. Mean (and standard error) arousal scores. *Difference is significant at $p<.05 .{ }^{* *}$ Difference is significant at $p<.005$.

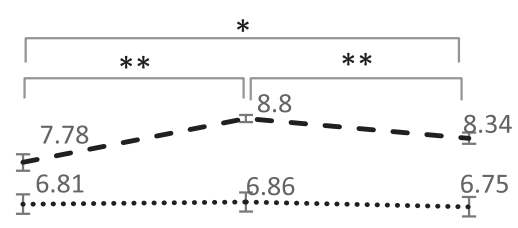

6

5

4

3

2

1

Phase 1

Phase 2

Phase 3

....... Control Group _ - - Jump Group

Figure 3. Mean (and standard error) valence scores. ${ }^{* *}$ Difference is significant at $p<.005$. 
Table 1. Mean raw scores (scale 1-9) and standard deviations for arousal and valence and results of the one-way ANOVA including group as a factor.

\begin{tabular}{|c|c|c|c|c|c|c|c|}
\hline & & \multicolumn{3}{|c|}{ Arousal } & \multicolumn{3}{|c|}{ Valence } \\
\hline & & Phase 1 & Phase 2 & Phase 3 & Phase 1 & Phase 2 & Phase 3 \\
\hline \multirow[t]{2}{*}{ Jump group } & $M$ & 4.83 & 7.58 & 4.47 & 7.78 & 8.80 & 8.34 \\
\hline & $S D$ & 1.96 & 2.11 & 2.25 & 1.26 & .57 & .77 \\
\hline \multirow[t]{2}{*}{ Control group } & $M$ & 3.59 & 4.08 & 4.31 & 6.81 & 6.86 & 6.75 \\
\hline & $S D$ & 1.42 & 1.94 & 1.99 & 1.37 & 1.33 & 1.34 \\
\hline \multirow[t]{3}{*}{ ANOVA } & $F$ & 9.94 & 56.18 & .12 & 10.79 & 72.95 & 37.59 \\
\hline & Sig & .002 & .000 & .731 & .002 & .000 & .000 \\
\hline & $\eta^{2}$ & .12 & .43 & .00 & .12 & .49 & .35 \\
\hline
\end{tabular}

were found between Phase 1 and Phase 3. In the control group, there was a significant difference between Phase 1 and Phase $2 \quad(F(1,35)=5.51$, $p=.025$, partial $\left.\eta^{2}=.14\right)$, and between Phase 1 and Phase $3\left(F(1,35)=8.09, p=.007\right.$, partial $\left.\eta^{2}=.19\right)$, but no differences were found between Phase 2 and Phase 3.

Moreover, in the jump group, the changes in valence were statistically significant between Phase 1 and Phase $2(F(1,34)=29.75, p<.001$, partial $\left.\eta^{2}=.47\right)$, between Phase 2 and Phase $3(F(1,34)=$ 9.68, $p=.004$, partial $\left.\eta^{2}=.22\right)$, and between Phase 1 and Phase $3\left(F(1,34)=8.00, p=.008\right.$, partial $\left.\eta^{2}=.19\right)$. For the control group, no significant differences were found between phases.

With respect to cognitive performance, Table 2 summarises the mean scores for all the tasks in each phase. The analysis of variance did not yield any significant difference between groups on any task throughout the study, and there was also no significant group $x$ phase interaction. However, to analyse in detail the trends and significant effects observed, a simple effects analysis was carried out in order to compare between phases within each group. This analysis revealed a trend consisting of an improvement in the performance of the jump group between Phase 1 and Phase 2 on most of the cognitive tasks, which was not observed in the control group.
The results of the simple effects analysis for each cognitive test performed in the study are described below.

\section{Digit span task}

Although the differences between Phase 1 and Phase 2 in the span measure in the jump group did not reach significance $(p=.06)$, the number of correctly recalled series showed a statistically significant increase in the score between Phase 1 and Phase 2 in the jump group $\left(F(1,39)=4.86, p=.033\right.$, partial $\left.\eta^{2}=.11\right)$. As shown in Figure 4, the difference between Phase 2 and Phase 3 and between Phase 1 and 3 was not significant in either group.

\section{Go/No-Go task}

No significant differences were observed in accuracy on the No-Go trials between phases, in either group. Regarding the RT for the Go trials, in the jump group the contrast revealed significant differences between Phases 1 and 2 (Phase $1>$ Phase 2; $F(1,38)=5.71$, $p=.02$, partial $\left.\eta^{2}=.13\right)$, and between Phases 1 and 3 (Phase $1>$ Phase $3 ; F(1,38)=12.16, p<.001$, partial $\left.\eta^{2}=.24\right)$, but no differences were found between Phase 2 and 3. In the control group the contrasts revealed significant differences between Phase 1 and Phase $2\left(F(1,36)=7.28, p=.011\right.$, partial $\left.\eta^{2}=.17\right)$, between Phase 2 and Phase $3(F(1,36)=5.62, p=.02$,

Table 2. Mean scores and standard deviations for all the cognitive tasks per group in each phase.

\begin{tabular}{|c|c|c|c|c|c|c|c|c|c|c|c|c|}
\hline & \multicolumn{4}{|c|}{ Phase 1} & \multicolumn{4}{|c|}{ Phase 2} & \multicolumn{4}{|c|}{ Phase 3} \\
\hline & \multicolumn{2}{|c|}{ Controls } & \multicolumn{2}{|c|}{ Jump Group } & \multicolumn{2}{|c|}{ Controls } & \multicolumn{2}{|c|}{ Jump Group } & \multicolumn{2}{|c|}{ Controls } & \multicolumn{2}{|c|}{ Jump Group } \\
\hline & $M$ & $S D$ & $M$ & $S D$ & M & $S D$ & $M$ & $S D$ & $M$ & $S D$ & $M$ & $S D$ \\
\hline IOWA Gambling TaskTask & -22.97 & 581.20 & -238.19 & 765.54 & 106.76 & 537.44 & 120.71 & 865.61 & 182.43 & 695.89 & 243.03 & 1028.11 \\
\hline Digit Span (Max span) & 4.97 & .87 & 4.71 & .90 & 4.92 & .97 & 4.98 & .92 & 4.83 & .94 & 4.88 & .76 \\
\hline $\begin{array}{l}\text { Digit Span (Correct } \\
\text { series) }\end{array}$ & 4.84 & 1.50 & 4.59 & 1.66 & 4.81 & 1.85 & 5.17 & 1.66 & 4.75 & 1.80 & 5.03 & 1.39 \\
\hline Go-No Go (RT) & 379.08 & 44.07 & 391.12 & 56.90 & 367.10 & 43.01 & 375.81 & 48.71 & 357.60 & 43.01 & 374.59 & 44.59 \\
\hline Go-No Go (Accuracy) & .93 & .09 & .93 & .08 & .93 & .08 & .92 & .08 & .94 & .09 & .92 & .07 \\
\hline
\end{tabular}




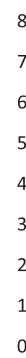

$$
\begin{aligned}
& \begin{array}{lll}
\text { Phase } 1 & \text { Phase } 2 & \text { Phase } 3
\end{array} \\
& \text {........ Control Group - - Jump Group }
\end{aligned}
$$

Figure 4. Mean (and standard error) number of correctly recalled series. *Difference is significant at $p<.05$.

partial $\left.\eta^{2}=.35\right)$, and between Phase 1 and Phase $3(F$ $(1,36)=23.88, p<.001$, partial $\left.\eta^{2}=.40\right)$. Figure 5 graphically represents the mean RT on the Go trials and the significant differences.

\section{lowa Gambling Task}

For the lowa Gambling Task, the within-subjects analysis of variance did not reveal statistical differences between phases in the control group. Conversely, the jump group significantly improved their net gain immediately after jumping (Phase 2) compared with Phase $1(F(1,32)=$ 4.54, $p=.041$, partial $\left.\eta^{2}=.12\right)$, which also increased between Phase 1 and $3(F(1,32)=4.49, p=.042$, partial $\eta^{2}=.12$ ). The mean net gain in each phase in the control and jump groups is shown in Figure 6.

\section{Discussion}

The aim of the present study was to assess whether a significant change in self-reported arousal and

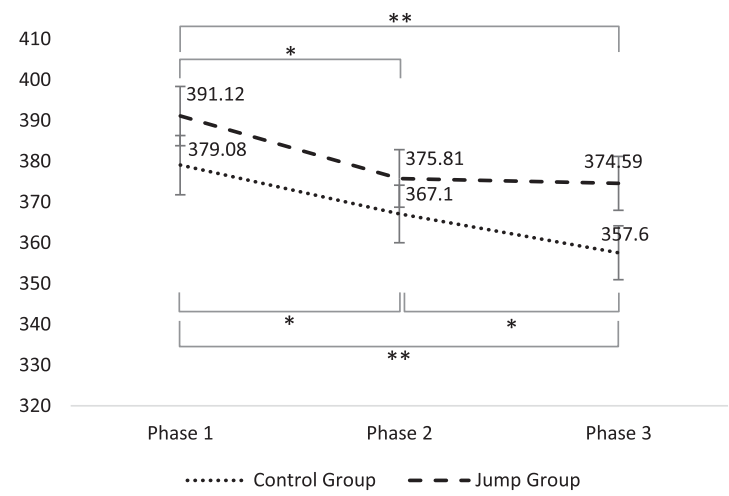

Figure 5. Mean RT (and standard error) on Go trials. *Difference is significant at $p<.05$. **Difference is significant at $p<.005$.

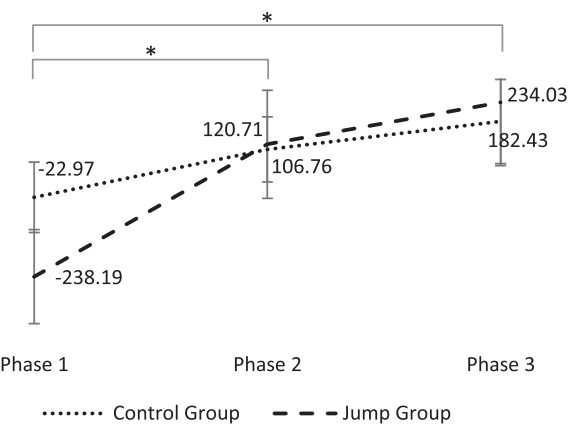

Figure 6. Mean net gain (and standard error) in the lowa Gambling Task. *Difference is significant at $p<.05$.

valence levels experienced during a high arousal situation - a $30 \mathrm{~m}$ bungee jumping free fall - affects information processing. To this end, we aimed to explore the impact of arousal and valence levels on cognitive tasks that assess working memory (reverse digit span), selective attention (Go/No-Go task), and decision-making (lowa Gambling Task). A group of bungee jumpers was assessed before and at two times following the jump, and their scores were compared against a control group of participants who did not jump. The control group was included in order to discard learning effects as a result of repeating the same task 3 times within a short period of time. The results obtained were rather clear-cut: The bungee jumping activity significantly increased the selfreported scores on the two emotional components (arousal and valence) in the jump group compared with the control group (except for arousal score at Phase 3, indicating recovery) with the jump group also showing the highest scores immediately after jumping, which indicates that the activity was able to induce acute changes. This, in turn, was accompanied by an improvement in some of the tasks, which will be described further. In particular, in the jump group arousal levels significantly increased in Phase 2, and returned to baseline at Phase 3 , while in the control group there was a sustained increase. However, given the very small effect sizes the latter was possibly due to an artefact or a mild increase in the level of alertness required to perform the tasks correctly. Valence scores were significantly higher in the jump group at all phases and although the levels dropped at Phase 3, they remained significantly higher than those observed in Phase 1, indicating an enduring effect on valence. These high positive valence ratings are worthy of further discussion, as they appear to be crucial for interpreting the present findings. 
Regarding working memory, participants in the jump group showed an overall improvement in the total number of correctly recalled series of digits, which returned to baseline in Phase 3, whilst for the control group no increases were observed across the phases. This pattern of findings confirms that the results found in this task are not attributable to learning processes. Both groups showed equivalent levels of performance in Phase 1, indicating that in the jump group working memory was not impaired prior to experiencing an arousing activity, contrary to the findings observed in previous studies (Idzikowski \& Baddeley, 1987; Leach \& Griffith, 2008). Although the testing time points of those studies are not directly comparable because they differ in the number of minutes participants were tested before jumping (30 min in our study, $10 \mathrm{~min}$ in Leach and Griffith's study and not specified in Idzikowski and Baddeley's), the three time points are similar in the sense that none of the studies tested participants very close to the time of jump for practical reasons. However, in our study, there were no differences in subjective arousal between Phase 1 and Phase 3 in the jump group, and valence was significantly higher (more positive) than in the control group at Phase 1. This pattern can be interpreted as a lack of self-reported anticipatory anxiety (characterised by high arousal and negative valence), which may explain why we did not find the impairment observed in the other two studies. Another striking finding is that performance actually improved after jumping, which is inconsistent with the reviewed studies that either found an impairment (Leach \& Griffith, 2008; Robinson et al., 2008), or no change in the functioning of both storage and processing components of working memory (Leach \& Ansell, 2008; Leach \& Griffith, 2008; Robinson et al., 2013) immediately after an extreme experience.

Selective attention, however, appeared to be unaffected by the activity, as both groups performed identically in all phases in terms of accuracy on the Go/No-Go task, a score that remained stable between phases. RT significantly decreased across phases, but this pattern of results was observed in both groups (except between Phase 2 and 3 in the jump group), and it therefore could be argued that this probably reflects a learning effect due to the nature of the task, which was relatively short and unchallenging. In any case, the acute increase in arousal and valence levels did not seem to have any impact on selective attention. In this regard, Leach and Griffith (2008) suggested that time is crucial in the sense that it can be an extra resource that helps to overcome cognitive impairments, and in line with the processing efficiency theory, individuals are able to perform at similar levels of effectiveness when compensatory resources, such as added time, are devoted to the task. This assumption implies a slowing down of the cognitive capacities and information processing, a trade off that has also been reported by Idzikowski and Baddeley (1987), who found a decrease in the speed of letter search but not in accuracy when participants were tested under high levels of anxiety. However, we failed to observe this pattern of performance in the Go/No-Go task.

Finally, regarding the lowa Gambling Task, our results suggest that, on the one hand learning effects can be ruled out since the net gain in the control group did not significantly increase across phases. On the other hand, the jump group obtained a significantly higher net gain immediately after jumping, although it could be argued that this finding is due to the fact that the scores obtained prior to the jump were so low that these could be taken to indicate an impairment that was not present after jumping. However, it should be noted that the differences between groups were not significant in Phase 1. Therefore, although we cannot conclude whether performance was actually improved by the activity or else impaired before the jump, the fact that the scores observed in the jump group at Phase 1 were considerably more negative prompts the suggestion that the participants most likely performed poorly, leading to more negative gains, which was resolved after the jump when values were almost identical to those of the control group. It is plausible to think that working memory capacity is taken up with thoughts related to the forthcoming activity at Phase 1, and, as suggested by Hinson, Jameson, and Whitney (2002), higher working memory loads during execution of the lowa Gambling Task interfere with the development of somatic markers to a higher extent, thus impairing decisionmaking.

Taken together, our results suggest that the bungee jumping activity - although proven to be capable of inducing acute changes in emotional responses - does not impair cognitive performance (at least in the tasks employed here), and can even lead to improvements. A free fall from a $30 \mathrm{~m}$ high bridge is a situation that can be perceived as potentially life-threatening and one that significantly increases arousal levels, as the organism interprets 
the situation as a state of emergency. The main difference between our study and previous studies conducted on emergency and stressful conditions relies on valence. As expected, due to the recreational nature of the activity, in all phases valence scores in the jump group were significantly higher than those in the control group, reaching a peak value immediately after the jump (almost at ceiling). This is in line with the rise in $\beta$-endorphins and enhanced euphoria ratings following a bungee jump, reported by Hennig et al. (1994). Therefore, valence could be the key factor, since previous studies in the literature have not evaluated this component, which means we cannot rule out the possibility that in these studies the participants experienced the stressors used as negative. The decline in cognitive abilities found in previous studies has traditionally been explained by the processing efficiency theory, which is based primarily on anxiety. Anxiety and arousal are two terms that are conceptually distinguishable and should not be used interchangeably, particularly since anxiety implies a negative valence and low dominance whilst an increase in arousal can be linked to both positive and negative valence. In the present study, the SAM questionnaire responses indicate that whilst self-reported arousal was very high, valence was very positive, and so anxiety, or arousal alone, cannot be put forward to explain the pattern of results obtained here. Conversely, positive valence combined with high arousal could be responsible for the improvement, or the lack of impairment, observed in these tasks. Although we expected some degree of moderation, that is, an attenuation of the negative impact of high arousal reported in the literature, we did not predict an actual improvement or a total lack of impairment.

In this regard, Fredrickson (2001) suggested the Broaden-and-Build theory, which emphasises the differential role of positive emotions. This theory states that positive emotions are capable of broadening thoughtaction repertoires and building personal resources (physical, intellectual, and psychosocial) that are necessary to cope with a given situation. These resources are supposed to be acquired under conditions in which a positive emotional state is experienced but also have the property of being durable. As predicted by the processing efficiency theory, a state of high arousal combined with a negative valence (anxiety) recruits resources, narrows attention, and impairs the central executive component of working memory, thus compromising performance.
The way to overcome this cognitive load is to mobilise auxiliary or compensatory resources and it is here where positive emotions might play a role. In this regard, it has been suggested that positive affect broadens the scope of cognition and action, making cognitive organisation more flexible, an effect that could be attributed to an increase in dopamine levels (Ashby, Isen, \& Turken, 1999). Fredrickson (2001) also states that positive emotions could mitigate the detrimental effects of negative emotions, speeding recovery from psychophysiological reactivity and helping the body return to baseline levels of arousal. The fact that in our study arousal levels returned to baseline at Phase 3 (which took place only 8 min after the jump) appears to provide support for this assumption. Taken together, the acute increase in valence found could have been strong enough to overcome the negative outcomes attributable to high arousal. Because arousal was accompanied by positive - and not negative - valence, we can say with confidence that anxiety was not present, and thus the expected impairments due to anxiety were not found. Conversely, a lack of impairment and even an improvement could be observed in the jump group. Future research should aim at directly comparing valence (positive vs. negative) with similar levels of arousal within a single study. Another future line of enquiry could include biological arousal markers such as cardiovascular reactivity or cortisol levels.

The results of the present study might be considered to have applied relevance in areas such as the emergency services (e.g. fire-fighters, ambulance drivers, civil protection personnel), in which professionals must behave and make rapid decisions under arousing conditions to ensure the survival of both themselves and others. Training these professionals to cope with emergency situations by enhancing and focusing on the positive emotions derived from their actions could improve - or at least not impair - their cognitive performance when facing threats.

\section{Acknowledgements}

We would like to thank La Sargantana, an adventure sports cooperative society based in Collbató, Barcelona, for granting us access to the sample of bungee jumping participants.

\section{Disclosure statement}

No potential conflict of interest was reported by the authors. 


\section{ORCID}

Judit Castellà (D) http://orcid.org/0000-0002-6094-3516

Jorge Luis Méndez-Ulrich (D) http://orcid.org/0000-0001-9718-0607

Antoni Sanz (D) http://orcid.org/0000-0002-7952-4477

\section{References}

Angrilli, A., Cherubini, P., Pavese, A., \& Manfredini, S. (1997). The influence of affective factors on time perception. Perception \& Psychophysics, 59(6), 972-982. doi:10.3758/BF03205512

Arnsten, A. F. (2009). Stress signalling pathways that impair prefrontal cortex structure and function. Nature Reviews Neuroscience, 10(6), 410-422.

Ashby, F. G., Isen, A. M., \& Turken, A. U. (1999). A neuropsychological theory of positive affect and its influence on cognition. Psychological Review, 106, 529-550. doi:10.1037/0033-295X. 106.3.529

Awh, E., Vogel, E. K., \& Oh, S. H. (2006). Interactions between attention and working memory. Neuroscience, 139(1), 201208. doi:10.1016/j.neuroscience.2005.08.023

Baddeley, A. D. (1986). Working memory. Oxford, UK: Oxford University Press.

Baddeley, A. D. (2007). Working memory, thought and action. Oxford, UK: Oxford University Press. doi:10.1093/acprof:oso/ 9780198528012.001.0001

Baddeley, A. D., Gathercole, S., \& Papagno, C. (1998). The phonological loop as a language learning device. Psychological Review, 105(1), 158-173.

Baddeley, A. D., \& Hitch, G. (1974). Working memory. In G. H. Bower (Ed.), The psychology of learning and motivation: Advances in research and theory (Vol. 8, pp. 47-89). New York: Academic Press. doi:10.1016/S0079-7421(08)60452-1

Bechara, A., Damasio, A. R., Damasio, H., \& Anderson, S. W. (1994). Insensitivity to future consequences following damage to human prefrontal cortex. Cognition, 50, 7-15. doi:10.1016/ 0010-0277(94)90018-3

Bradley, M., \& Lang, P. J. (1994). Measuring emotion: The selfassessment manikin and the semantic differential. Journal of Behavior Therapy and Experimental Psychiatry, 25(I), 49-59. doi:10.1016/0005-7916(94)90063-9

Brewer, M. (2000). Research design and issues of validity. In $\mathrm{H}$. Reis \& C. Judd (Eds.), Handbook of research methods in social and personality psychology (pp. 3-16). Cambridge: Cambridge University Press.

Castellà, J., Cuello, C., \& Sanz, A. (2017). Does time fly 20m above the ground? Exploring the role of affective response on time perception in a high-risk sport. Applied Cognitive Psychology, 31(6), 644-652. doi:10.1002/acp.3367

Coombes, S. A., Higgins, T., Gamble, K. M., Cauraugh, J. H., \& Janelle, C. M. (2009). Attentional control theory: Anxiety, emotion, and motor planning. Journal of Anxiety Disorders, 23, 1072-1079. doi:10.1016/j.janxdis.2009.07.009

Damasio, A. (1998). The somatic marker hypothesis and the possible functions of the prefrontal cortex. In The prefrontal cortex: Executive and cognitive functions (pp. 36-50). New York: Oxford University Press. doi:10.1093/acprof:oso/9780198524410. 003.0004

Engle, R. W., Kane, M. J., \& Tuholski, S. W. (1999). Individual differences in working memory capacity and what they tell us about controlled attention, general fluid intelligence, and functions of the prefrontal cortex. In A. Miyake \& P. Shah (Eds.), Models of working memory (pp. 102-134). New York: Cambridge University Press.

Eysenck, M. W., \& Calvo, M. G. (1992). Anxiety and performance: The processing efficiency theory. Cognition \& Emotion, 6(6), 409-434. doi:10.1080/02699939208409696

Eysenck, M. W., \& Derakshan, N. (2011). New perspectives in attentional control theory. Personality and Individual Differences, 50, 955-960. doi:10.1016/j.paid.2010.08.019

Eysenck, M. W., Derakshan, N., Santos, R., \& Calvo, M. G. (2007). Anxiety and cognitive performance: Attentional control theory. Emotion, 7(2), 336-353. doi:10.1037/1528-3542.7.2. 336

Fenz, W. D., \& Epstein, S. (1967). Gradients of physiological arousal in parachutists as a function of an Approaching jump. Psychosomatic Medicine, 29(1), 33-51.

Fredrickson, B. L. (2001). The role of positive emotions in positive psychology. The broaden-and-build theory of positive emotions. The American Psychologist, 56(3), 218-226. doi:10. 1037/0003-066X.56.3.218

Geuss, M., Stefanucci, J., de Benedictis-Kessner, J., \& Stevens, N. (2010). A balancing act: Physical balance, through arousal, influences size perception. Attention, Perception, \& Psychophysics, 72(7), 1890-1902. doi:10.3758/APP.72.7.1890

Hennig, J., Laschefski, U., \& Opper, C. (1994). Biopsychological changes after bungee jumping: $\beta$-endorphin immunoreactivity as a mediator of euphoria? Neuropsychobiology, 29, 28-32. doi:10.1159/000119059

Hinson, J. M., Jameson, T. L., \& Whitney, P. (2002). Somatic markers, working memory, and decision making. Cognitive, Affective, \& Behavioral Neuroscience, 2, 341-353.

Idzikowski, C., \& Baddeley, A. D. (1987). Fear and performance in novice parachutists. Ergonomics, 30(10), 1463-1474. doi:10. 1080/00140138708966039

Jonides, J. (1995). Working memory and thinking. In E. E. Smith, \& D. N. Osherson (Eds.), Invitation to cognitive Science: Thinking (Vol. 3, 215-265). Cambridge, MA: MIT Press.

Leach, J., \& Ansell, L. (2008). Impairment in attentional processing in a field survival environment. Applied Cognitive Psychology, 22(5), 643-652. doi:10.1002/acp.1385

Leach, J., \& Griffith, R. (2008). Restrictions in working memory capacity during parachuting: A possible cause of "no pull" fatalities. Applied Cognitive Psychology, 22(2), 147-157. doi:10.1002/acp.1364

Presentation ${ }^{\circledast}$. (2018). Version 18.0 [Computer software]. Berkeley, CA: Neurobehavioral Systems Inc. (www.neurobs. com).

Robinson, S. J., Leach, J., Owen-Lynch, P. J., \& Sünram-Lea, S. I. (2013). Stress reactivity and cognitive performance in a simulated firefighting emergency. Aviation Space and Environmental Medicine, 84(6), 592-599. doi:10.3357/ASEM.3391.2013

Robinson, S. J., Sünram-Lea, S. I., Leach, J., \& Owen-Lynch, P. J. (2008). The effects of exposure to an acute naturalistic stressor on working memory, state anxiety and salivary cortisol concentrations. Stress, 11(2), 115-124. doi:10.1080/ 10253890701559970

Ruffman, T. (2011). Ecological validity and age-related change in emotion recognition. Journal of Nonverbal Behavior, 35(4), 297-304. doi:10.1007/s10919-011-0116-3 
Russell, J. A. (1980). A circumplex model of affect. Journal of Personality and Social Psychology, 39, 1161-1178. doi:00223514/80/3906-1161\$00.75

Salthouse, T. A. (1992). Why do adult age differences increase with task complexity? Developmental Psychology, 28, 905-918.
Shadish, W., Cook, T., \& Campbell, D. (2002). Experimental and quasi-experimental designs for generalized causal inference. Boston: Houghton Mifflin.

Ziems, D., \& Christman, S. (1998). Effects of mood on color perception as a function of dimensions of valence and arousal. Perceptual and Motor Skills, 87(2), 531-535. doi:10.2466/pms.1998.87.2.531 